Revista

Ibero-Americana

de Estratégıa

\title{
UM SISTEMA DE GESTÃO DO CONHECIMENTO COMO FOMENTADOR DE REDES
} ESTRATÉGICAS INTERORGANIZACIONAIS

A KNOWELEDGE MANAGEMENT SYSTEM AS A MECHANISM TO FOMENT INTERORGANIZATIONAL NETWORKS

UN SISTEMA DE GESTIÓN DEL CONECIMIENTO COMO FOMENTADOR DE REDES ESTRATEGICAS INTERORGANIZACIONALES

\section{Mário José Batista Franco}

Professor no Departamento de Gestão e Economia da Universidade da Beira Interior - UBI E-mail: mfranco@ubi.pt (Portugal)

\section{Marisa Regina Reduto Santos Barbeira}

Professora na Escola Superior de Saúde Dr. Lopes Dias do Instituto Politécnico de Castelo Branco E-mail: marisabarbeira@ esald.ipcb.pt (Portugal) 
UM SISTEMA DE GESTÃO DO CONHECIMENTO COMO FOMENTADOR DE REDES ESTRATÉGICAS INTERORGANIZACIONAIS

\title{
RESUMO
}

O conhecimento é um recurso valioso numa organização. Neste sentido, as redes, dentro e entre organizações, como estratégia para a partilha do conhecimento, podem ser uma das vias mais importantes que uma organização pode adoptar. As diferentes organizações, ao estabelecerem relacionamentos com outras organizações, têm a possibilidade de fomentar e partilhar o conhecimento que, estrategicamente, serve como um mecanismo de produtividade, eficiência e excelência organizacional. O objectivo deste artigo é desenvolver um suporte teórico que combine diferentes conceitos e elementos para explicar e compreender o fenómeno das redes estratégicas nas organizações, como um mecanismo de partilha do conhecimento. É ainda proposto um modelo conceptual de análise intra e entre organizações, evidenciando o sistema de gestão do conhecimento como fomentador das redes interorganizacionais e com vista à partilha de conhecimento organizacional.

Palavras-chave: Redes Interorganizacionais; Gestão do Conhecimento; Estratégia Corporativa; Modelo.

\section{A KNOWELEDGE MANAGEMENT SYSTEM AS A MECHANISM TO FOMENT INTERORGANIZATIONAL NETWORKS}

\begin{abstract}
The knowledge is a valuable resource in an organization .In this sense, the networks, as a strategy for the sharing of knowledge, can be one of the most important assets that an organization can adopt. Different organizations, to establish relationships with other organizations, have the opportunity to promote and share the knowledge that, strategically, serves as a mechanism for productivity and organizational effectiveness. The objective of this article is to develop a theoretical support that combines different concepts and elements to explain and understand the phenomenon of strategic alliances in organizations, as a mechanism for sharing of knowledge. We also propose a conceptual model of analysis both within and between organizations, in order to emphasize importance of knowledge management system to foment interorganizational networks and for the sharing of organizational knowledge.
\end{abstract}

Keywords: Interorganizational Networks; Knowledge Management; Corporate Strategy; Model.

Revista Ibero-Americana de Estratégia - RIAE, São Paulo, v. 8, n. 2, p. 04-30, jul./dez. 2009. 
Mário José Batista Franco \& Marisa Regina Reduto Santos Barbeira

\section{UN SISTEMA DE GESTIÓN DEL CONECIMIENTO COMO FOMENTADOR DE REDES ESTRATEGICAS INTERORGANIZACIONALES}

\section{RESUMEN}

El conocimiento es uno recurso valioso en la organización. Asín, las redes en y entre organizaciones, como estrategia para el intercambio del conocimiento, pueden ser una de las vías más importantes que una organización puede adoptar. Las diferentes organizaciones, estableciendo relacionamientos con otras organizaciones, tienen la posibilidad de fomentar y compartir el conocimiento que, estratégicamente, es como uno mecanismo de productividad, eficiencia y excelencia organizacional. El objetivo de este trabajo es desarrollar un suporte teórico que combine diferentes conceptos y elementos para explicar y comprender el fenómeno de las redes estratégicas en las organizaciones, como un mecanismo de intercambio del conocimiento. Es tanbien propuesto uno modelo conceptual de análisis intra y entre organizaciones, evidenciando un sistema de gestión del conocimiento como fomentador de las redes inter-organizacionales y con vista al partilla del conocimiento organizacional.

Palabras-clave: Redes Inter-organizacionales; Gestión del Conocimiento; Estrategia Corporativa; Modelo. 


\section{INTRODUÇÃO}

Em função das condições do meio envolvente - incerteza, complexidade e globalização - e para se tornarem competitivas, as organizações deverão adoptar as estratégias que resultem mais eficazes (Franco, 2001). Não parecem subsistir muitas dúvidas acerca do crescente alargamento dos mercados. Segundo Parkhe, Wasserman e Ralston (2006), a elevada e profunda revolução em termos económicos, financeiros, tecnológicos e as variações na composição da procura, entre outros aspectos, alteram, cada vez mais, as estruturas das organizações, diluindo as fronteiras e tornando o mundo cada vez mais pequeno. Isso significa que as organizações têm de competir num mercado mais aberto no qual os níveis de exigência serão maiores (Macnabb \& O’Neill, 1996). Como referem ainda Murray (1995) e Márquez-García, Hernández-Ortiz, Vallejo-Martos e Cañas-Lozano (1998), a crescente globalização dos mercados, o aumento da concorrência, a velocidade e a complexidade das mudanças tecnológicas conduzem as organizações para uma abertura das suas fronteiras, passando, assim, de uma forma tradicionalmente estática e de mutação lenta para uma concepção mais dinâmica e adaptável.

Nesse contexto, as redes estratégicas interorganizacionais podem ser vistas como um mecanismo que permite às organizações alcançar os seus objectivos. De facto, num meio envolvente incerto e turbulento, as redes podem assumir-se como uma estratégia adequada, já que podem melhorar a competitividade das organizações envolvidas (Inkpen \& Beamish, 1997). Contractor e Lorange (1988) argumentam também que uma estratégia bem-sucedida muitas vezes requer a adopção de redes que, segundo Kogut (1988), Mason (1993), Morrison e Mezentseff (1997) e Stafford (1994), permitem às organizações obter vantagens competitivas.

O pressuposto básico da estratégia de redes é que uma determinada organização depende dos recursos controlados por outra e, por esta via, as organizações têm acesso a esses recursos externos através da sua posição na rede (Johanson \& Mattsson, 1987). Assim, neste estudo, uma rede interorganizacional é definida como um meio através do qual os actores intervenientes na rede conseguem aceder a uma maior variedade de recursos inatingíveis detidos por outros actores.

Nesse segmento, a posição estratégica de uma organização não depende, exclusivamente, da quantidade e da qualidade dos recursos que possui, mas sim da posição que assume no seio da rede de relacionamentos em que se insere, dado que é a partir dessas redes interorganizacionais que pode aceder, de forma directa ou indirecta, às competências essenciais para o desenvolvimento da sua actividade (Suire \& Vicente, 2008).

Revista Ibero-Americana de Estratégia - RIAE, São Paulo, v. 8, n. 2, p. 04-30, jul./dez. 2009. 
Assim, as redes são vistas como uma estratégia que permite às organizações dispor dos necessários recursos, bem como explorar oportunidades de mercado que não conseguiriam alcançar de uma forma isolada (Zinn, Proença \& Rosko, 1997).

São várias as razões por que as organizações optam por redes estratégicas: reduzir os custos de desenvolvimento tecnológico ou de entrada no mercado; reduzir os riscos de desenvolvimento ou de entrada no mercado; conseguir economias de escala na produção; reduzir o tempo de desenvolvimento e comercialização de novos produtos (Franco, 2001, 2003; Tidd, Bessant \& Pavitt, 2003); e acompanhar o rápido progresso e difusão das tecnologias associadas ao novo paradigma técnico-económico, baseado nas tecnologias da informação (Cozzarin \& Percival, 2006; Forbes \& Wield, 2008; Huang \& Chang, 2008; Percival \& Cozzarin, 2008; Suire \& Vicente, 2008; Weber \& Khademian, 2008).

Por outro lado, as redes estratégicas podem também ser entendidas como um mecanismo de aprendizagem, transferência e de partilha de conhecimentos dentro e entre organizações, bem como forma de controlar este importante recurso que é o conhecimento (Cassiman \& Veugelers, 2002; Cozzarin \& Percival, 2006; Fritsch, 2004; Lorenz, 1992; Schmiedeberg, 2008; Suire \& Vicente, 2008; Weber \& Khademian, 2008; Yeung, 1994). Estabelecer relações entre organizações permite transferir, partilhar e disseminar conhecimento e, ainda, dinamizar um conjunto de competências de aprendizagem organizacional, com vista a reforçar e a alcançar a competitividade da organização (Azevedo, 2000).

As redes interorganizacionais criam e desenvolvem novo conhecimento, para isso, porém, tem de existir um sistema de gestão do conhecimento para fomentar esse tipo de relacionamento. Nesse sentido, a gestão do conhecimento entende-se como um processo colectivo, de natureza interactiva, que pressupõe uma partilha de informação e atitudes nas várias fases do desenvolvimento do conhecimento. A gestão do conhecimento será um processo sistemático, articulado e intencional, apoiado na criação, codificação, disseminação e apropriação de conhecimentos, cujo objectivo será atingir a excelência organizacional (Fliaster \& Spiess, 2008; Franco \& Mariano, 2007; Suire \& Vicente, 2008; Weber \& Khademian, 2008).

Nesse segmento, torna-se necessário um conhecimento mais aprofundado desse tipo de processo de relacionamento entre organizações, em particular, numa perspectiva estratégica de relacionamento através da gestão do conhecimento. Neste trabalho pretende-se desenvolver um suporte teórico que combine diferentes conceitos e elementos para explicar e compreender o fenómeno das redes estratégicas nas organizações, como um mecanismo de partilha do conhecimento. Mais precisamente, pretende-se mostrar a importância da gestão estratégica do

Revista Ibero-Americana de Estratégia - RIAE, São Paulo, v. 8, n. 2, p. 04-30, jul./dez. 2009. 
conhecimento entre diferentes organizações e, para este fim, será proposto um modelo conceptual de análise que possa servir de contribuição para estudos empíricos nesta área do conhecimento.

\section{ENQUADRAMENTO TEÓRICO: REVISÃO DA LITERATURA}

\subsection{REDES INTERORGANIZACIONAIS: CARACTERÍSTICAS E TIPOLOGIA}

Embora seja observado o seu recente interesse no campo organizacional, o termo "rede" não é novo e apresenta diversos significados e aplicações nos mais variados contextos, já há algum tempo. A abordagem das redes data dos anos trinta nas investigações sobre as organizações, apesar da génese e origem dos conceitos também dever muito às disciplinas de sociologia e antropologia (Hoang \& Antoncic, 2003; Parkhe et al., 2006).

No campo de estudos das ciências sociais, o termo "rede" designa um conjunto de pessoas ou organizações interligadas directa ou indirectamente (Faggion, Balestrin \& Weyh, 2002). Interacção, relacionamento, aliança, acordo, cooperação, redes, entre outros, são termos habituais e utilizados na dimensão organizacional. A sua aplicação prática é que poderá estabelecer as diferentes formas de relacionamento entre organizações (Franco, 2001).

As investigações sobre redes podem envolver o estudo de um conjunto de factores e aspectos, como o tamanho da rede, a estrutura, o processo interactivo, a influência, o comportamento e as capacidades (Coviello, 2006). Com efeito, a literatura é consensual em reconhecer que uma rede estratégica ocorre quando duas ou mais organizações decidem conjugar esforços para perseguir um objectivo comum (Aaker, 1992; Johanson \& Mattsson, 1987; Lorange \& Roos, 1996). Nesse caso, as partes envolvidas procuram desenvolver uma vantagem cooperativa que tenha efeitos positivos sobre o seu desempenho individual e colectivo.

Redes de cooperação e redes estratégicas são diferentes termos para uma mesma realidade. No entender de outros autores (e.g., Faulkner, 1992), uma rede estratégica é uma forma de organização das actividades dos parceiros que envolve um maior grau de integração do que outras formas de cooperação.

Segundo Teece (1998), uma rede estratégica implica algum grau de coordenação estratégica e operacional das actividades realizadas pelas organizações. Dussauge e Garrete (1995) referem que as redes estratégicas são projectos de colaboração implantados por empresas rivais, operando no mesmo sector. As organizações mantêm, entretanto, a sua independência. Essa definição exclui, desse modo, fusões e aquisições, as quais levam à perda de autonomia de pelo menos um parceiro.

Revista Ibero-Americana de Estratégia - RIAE, São Paulo, v. 8, n. 2, p. 04-30, jul./dez. 2009. 
Mário José Batista Franco \& Marisa Regina Reduto Santos Barbeira

De acordo com Häkansson e Ford (2002), o conceito de redes interorganizacionais significa a interacção, o dinamismo, a confiança, a adaptação e o relacionamento existente entre e dentro das organizações. Assim, existe um conjunto de variáveis essenciais que interagem entre si, as quais permitem o tal relacionamento estratégico, que conduz a determinados benefícios.

Eiriz (2004) refere que as redes estratégias correspondem a uma decisão estratégica adoptada por duas ou mais organizações independentes (nacionais ou estrangeiras) com vista a trocar ou partilhar recursos para procurar oportunidades de mercado e alcançar benefícios mútuos. Esse conceito incorpora, claramente, o processo de interacção no quadro do relacionamento entre organizações, definido por Häkansson e Snehota (1989) como a necessidade da adaptação, da cooperação e do conflito, da interacção social e da rotina.

Outros autores (Hax \& Majluf, 1988; Johnson \& Scholes, 1999), ao distinguirem decisões estratégicas e decisões operacionais, entendem que as diferenças entre uma rede estratégica e outros tipos de relação de cooperação residem fundamentalmente no facto das primeiras reunirem um conjunto de características que lhes conferem uma dimensão estratégica que não está presente nas segundas. Essas características são as seguintes: (1) uma rede estratégica resulta de um conjunto coerente de decisões; (2) trata-se de um meio para desenvolver uma vantagem competitiva sustentável; (3) tem um impacto organizacional de longo prazo; (4) é um meio para responder a oportunidades e ameaças externas; (5) baseia-se em recursos organizacionais que mostram forças e fraquezas; (6) afecta decisões operacionais; (7) envolve todos os níveis hierárquicos da organização; (8) é influenciada pelo seu contexto cultural e político; e (9) envolve, directa ou indirectamente, todas as actividades da organização. Existem, assim, várias características numa rede que, quando se verificam de forma simultânea, fazem com que ela se torne estratégica.

As redes interorganizacionais podem ser classificadas a partir da observação de quatro elementos-chave (cooperação, hierarquia, contrato e conivência), possibilitando, dessa forma, uma ampla variedade de tipologias (Faggion et al., 2002). Tal como referem Inkpen e Tsang (2005), existem diferentes tipos de estratégia, entre as quais se destacam as redes interorganizacionais.

São vários os tipos de redes estratégicas presentes na literatura. Faulkner (1992) apresenta três dimensões de classificação das redes estratégicas e, em função das diferentes conjugações dessas dimensões, extrai oito opções. Na primeira dimensão (focalizada versus complexa), o autor socorre-se das actividades desenvolvidas pelos parceiros; a segunda dimensão ("joint venture" versus "non-joint venture") respeita ao capital e forma jurídica da rede; na terceira dimensão (dois parceiros versus consórcio) sobressai o número de parceiros envolvidos.

Revista Ibero-Americana de Estratégia - RIAE, São Paulo, v. 8, n. 2, p. 04-30, jul./dez. 2009. 
Por seu lado, Root (1988) utiliza duas dimensões distintas das anteriores para classificar as redes estratégicas internacionais. Essas dimensões são a nacionalidade das organizações e o tipo de cooperação desenvolvida. No primeiro caso, os acordos podem ser uninacionais (feitos para um único país), binacionais (dois países) ou multinacionais (vários países). No segundo caso, as redes podem constituir-se como transacções em mercado. Yoshino e Rangan (1995) desagregam as ligações interorganizacioinais em função do tipo de contratos (tradicionais e não tradicionais) e do grau de envolvimento de capital (fusões e aquisições, e criação ou não criação de uma nova entidade).

Para estes autores, as redes estratégicas são ligações interorganizacionais que envolvem contratos não tradicionais (incluem neste caso vários acordos baseados em actividades como, por exemplo, produção ou investigação e desenvolvimento conjunto) ou arranjos de capital, excluindo as fusões e aquisições, e as joint ventures que são subsidiárias de multinacionais. Dessa forma, excluem do âmbito das redes estratégicas acordos como o licenciamento e franshising que são considerados contratos tradicionais.

De sublinhar ainda que, segundo Eiriz (2004), as redes estratégicas são consideradas uma importante forma de criar oportunidade e transferir conhecimento, pois a formação de extensas redes de relacionamento possibilitam, directa ou indirectamente, ter uma maior percepção do meio envolvente. A relação entre organizações sustentada numa rede de criação e partilha de conhecimento baseada na interdependência entre as partes e na reciprocidade de custos e benefícios é uma forma de rede estratégica.

Reconhecendo este facto, criar redes de conhecimento, implica determinar quem tem acesso ao conhecimento, como é gerido, como é disseminado e que ferramentas a adoptar no intuito de auxiliar a transferência do conhecimento de forma estratégica (Teece, 2000). Assim, é necessário perceber de que forma o conhecimento pode ser um mecanismo estratégico fundamental para as organizações.

\subsection{GESTÃO ESTRATÉGICA E CONHECIMENTO NAS ORGANIZAÇÕES}

Estudos realizados por Mintzberg, Ahlstrand e Lampel (2000) definem estratégia como uma ferramenta imprescindível para as organizações. Segundo a visão de Grant (1991), estratégia pode ser definida como uma adaptação realizada por uma organização entre os seus recursos internos, habilidades, oportunidades e riscos criados pelo ambiente externo.

Revista Ibero-Americana de Estratégia - RIAE, São Paulo, v. 8, n. 2, p. 04-30, jul./dez. 2009. 
Mário José Batista Franco \& Marisa Regina Reduto Santos Barbeira

Outros investigadores (Thompson Jr. \& Strickland III, 2000) referem que a estratégia de uma organização consiste num conjunto de mudanças competitivas e abordagens comerciais, que os gerentes executam para atingirem o melhor desempenho da organização, de forma a reforçar a posição da mesma no mercado, promover a satisfação dos clientes e atingir os objectivos de desempenho.

Segundo Hitt, Ireland e Hoskisson (2001), a estratégia é definida como um processo que permite combinar as condições de um mercado em constante transição com a estrutura competitiva dos recursos, capacidades e competências de uma organização, também, em constante transição.

Por outro lado, de acordo com Teece (1998), a gestão estratégica representa o processo através do qual as organizações obtêm e sustentam vantagens competitivas, ou seja, corresponde às decisões e acções necessárias para que uma empresa alcance a competitividade. Ainda segundo o mesmo autor, a definição de gestão estratégica consiste em acordos nos quais dois ou mais parceiros dividem o compromisso de alcançar um objectivo comum, unindo todas as suas capacidades e recursos e coordenando as suas actividades.

As organizações utilizam tanto os bens tangíveis quanto os intangíveis no desenvolvimento e implementação de estratégias, no entanto, os bens intangíveis são mais adequados à produção da vantagem competitiva entre as organizações (Hitt et al., 2001). Assim, o processo associado ao conceito de estratégia apresenta um carácter dinâmico, mediante acções, como a formulação e implementação da estratégia e, consequentemente, obtenção de resultados.

O poder económico e de produção de uma organização moderna está mais concentrado nos activos intelectuais (intangíveis) do que nos seus recursos materiais (Drucker, 1993; Quinn, 1996). Por exemplo, no início da década de 1990, Drucker (1993) afirmava que a produtividade do conhecimento seria o factor determinante da posição competitiva de uma organização, de uma indústria e de todo um país. Alguns anos depois, Davenport e Prusak (1998) verificaram que as actividades baseadas no conhecimento e voltadas para o desenvolvimento de produtos e processos eram as principais funções internas das organizações. Na mesma época, Nonaka e Takeuchi (1997) evidenciaram que o conhecimento das organizações era a sua fonte de vantagem competitiva.

A gestão do conhecimento, vista como um conjunto de processos que governa a criação, a disseminação e a utilização do conhecimento para atingir plenamente os objectivos da organização, é uma nova área na confluência entre a tecnologia da informação e da gestão, um novo campo entre a estratégia, a cultura e os sistemas de informação de uma organização.

Com a gestão do conhecimento começa-se a rever a organização, as suas estratégias, a sua estrutura e a sua cultura. Segundo Loureiro (2003), para transformar o conhecimento em activo

Revista Ibero-Americana de Estratégia - RIAE, São Paulo, v. 8, n. 2, p. 04-30, jul./dez. 2009. 
organizacional com valor, o conhecimento, a experiência e a perícia devem ser formalizados, distribuídos e aplicados. A gestão do conhecimento é considerada a parte fundamental da estratégia para usar conhecimento na criação de vantagens competitivas sustentáveis na actual envolvente organizacional (Bloodgood \& Salisbury, 2001; Grant 2006; Gray \& Meister, 2006; Loureiro, 2003; Sher \& Lee, 2004).

Nesse sentido, pode-se conceituar a gestão do conhecimento como:

\begin{abstract}
um conjunto de estratégias que visa colocar o conhecimento (tanto tácito quanto explícito) em acção, através de sistemas e processos que possibilitem às pessoas contribuírem para o conhecimento colectivo da empresa e dele retirarem o que necessitam para o seu desenvolvimento e, ao mesmo tempo, para o aperfeiçoamento das operações organizacionais (Moura, 2001, p. 1).
\end{abstract}

Na prática, "a gestão do conhecimento consiste na identificação e no mapeamento dos activos de conhecimento da organização, divulgando e gerando novos conhecimentos para a vantagem competitiva e partilhando as melhores práticas e tecnologias que impulsionarão estes processos" (Serrano \& Fialho, 2003, p. 126). Logo, as actividades principais para a gestão do conhecimento, de acordo com Franco e Mariano (2007) e Silva e Neves (2003), prendem-se com a geração (criação ou aquisição), codificação (ou armazenamento) e transferência do conhecimento.

Nesse contexto, um possível mecanismo formal de prospecção da informação para a sustentação da gestão do conhecimento e das directrizes da rede estratégica é a inteligência competitiva. Ao incorporar o conceito de estratégia, os teóricos da Administração trouxeram também o conceito de Inteligência (Balestrin \& Vargas, 1998). Um sistema de inteligência deve em primeiro lugar identificar os principais tipos de informações competitivas e as melhores fontes dessas informações. Simultaneamente, deve avaliar a validade e confiabilidade da informação, interpretá-la e organizá-la de forma apropriada e, finalmente, enviar as melhores informações para os diferentes membros da organização.

A questão da inteligência competitiva tem vindo a crescer com muita intensidade. A estruturação e a operação de um sistema de informação que organize a prática de recolha e análise de informações têm vindo a ser evidenciadas como uma preocupação importante nas organizações, pelo facto de todo o fluxo de informações ser necessário para a tomada de decisões. Torna-se necessário o máximo de empenho dos dirigentes para identificar as informações relevantes e de grande interesse para a organização, que, em geral, não surgem espontaneamente (Lesca, Freitas \& Cunha, 1996).

Revista Ibero-Americana de Estratégia - RIAE, São Paulo, v. 8, n. 2, p. 04-30, jul./dez. 2009. 
Mário José Batista Franco \& Marisa Regina Reduto Santos Barbeira

Sendo assim, existe a necessidade da organização utilizar um processo sistemático e formal, que retire de um manancial de informações aquelas que sejam críticas ao processo de decisão. De facto, este objectivo pode ser alcançado através de um sistema de "inteligência competitiva".

\section{SISTEMA DE GESTÃO DO CONHECIMENTO EM REDES INTERORGANIZACIONAIS}

\subsection{GESTÃO ESTRATÉGICA DO CONHECIMENTO ORGANIZACIONAL}

O conhecimento é uma acção própria ou natural de um comportamento ou percepção. Nonaka e Takeuchi (1997) consideram o conhecimento como um processo humano dinâmico de justificar a crença pessoal em relação à verdade. Polanyi (1966) citado por Sveiby (1998), afirma que o conhecimento é uma actividade que reflecte o processo de saber. É provável que "o processo humano de saber seja criado pela natureza para nos ajudar a sobreviver num ambiente quase sempre hostil" (Sveiby, 1998, p. 44). Assim, o conhecimento é "uma mistura fluida de experiência condensada, valores, informação contextual, a qual proporciona uma estrutura para a avaliação e incorporação de novas experiências e informações” (Davenport \& Prusak, 1998, p. 6).

Segundo Fleury e Oliveira (2001), o conhecimento organizacional é definido como o conjunto compartilhado de crenças sobre relações causais mantidas por indivíduos dentro de um grupo. Assim, o conhecimento das organizações está nas percepções, crenças e valores que um indivíduo e uma organização tomam como certas ao longo do seu ciclo de desenvolvimento.

De acordo com Nadler e Tushman (2000), com os avanços na área da tecnologia de informação, a capacidade de criar negócios foi alterada de forma irreversível. A economia passou a valorizar mais os trabalhadores portadores de conhecimento. Nesse sentido, a necessidade de gerir estrategicamente o conhecimento cresceu a partir do reconhecimento de que as estruturas organizacionais inflexíveis não conseguiam competir perante a mudança nos mercados (Soo, 2002). Zack (1999) reforça esta ideia ao referir que a essência da gestão estratégica do conhecimento reside na identificação dos recursos baseados no conhecimento.

Fleury e Oliveira (2001), por sua vez, acreditam que o interesse por uma abordagem estratégica baseada em recursos, como o conhecimento, pode ser entendida como uma mais-valia para as organizações estabelecerem resultados superiores relativamente a outras organizações.

Revista Ibero-Americana de Estratégia - RIAE, São Paulo, v. 8, n. 2, p. 04-30, jul./dez. 2009. 
Nesse contexto, a gestão estratégica do conhecimento permite analisar o quanto efectivo é a criação de novos produtos e serviços que incorporam o conhecimento da organização. Está relacionada com as actividades de avaliação, construção, aquisição, aplicação, retenção e partilha de conhecimento (McCann \& Buckner, 2004).

Poucas abordagens são encontradas na literatura relativamente ao conceito e explicação da gestão estratégica do conhecimento. Na maioria das vezes, examina-se de forma isolada a gestão do conhecimento, baseada nos recursos estratégicos das organizações. Para colmatar esta lacuna, seguidamente serão apresentadas as principais abordagens presentes na literatura relativas à gestão estratégica do conhecimento.

Segundo Hansen, Nohria e Tierney (1999), existem dois tipos de estratégia para a gestão do conhecimento: a codificada e a personalizada. A estratégia codificada está relacionada com o conhecimento codificado e armazenado numa base de dados, podendo, facilmente, ser utilizado por qualquer membro da organização. Por outro lado, a estratégia personalizada ocorre quando o conhecimento é disseminado através da proximidade entre as pessoas, as quais o desenvolvem e partilham através de contactos directos de pessoa para pessoa.

A escolha da melhor estratégia, de acordo com os mesmos autores, depende dos seguintes aspectos: (1) se a organização trabalha com um produto inovador ou não; e (2) se as pessoas que resolvem os problemas possuem habilidades para lidar com o conhecimento tácito ou explícito. Embora Hansen et al. (1999) tenham realizado uma análise de cada estratégia, eles afirmam que as organizações deveriam optar pelas duas estratégias porque ambas são complementares.

De acordo com Krogh (1998), a escolha de uma estratégia deve basear-se no equilíbrio entre o conhecimento existente e o novo, bem como em processos centrais do conhecimento e nas metas da organização. Estes autores identificaram a expansão, a investigação e a apropriação como estratégias do conhecimento. A estratégia de apropriação está relacionada com a transferência de novos conhecimentos. Tem como principal objectivo a construção de um domínio novo do conhecimento por meio da transferência de conhecimento a partir de fontes externas. A estratégia de expansão examina o processo de criação do conhecimento, a partir do conhecimento existente. Por último, a estratégia de investigação foca a criação de novos conhecimentos, em que a equipa tem a responsabilidade máxima na construção de novo conhecimento.

Ainda segundo os referidos autores, o desenvolvimento de vantagens baseadas no conhecimento requer uma adequada atenção e ao mesmo tempo um desenvolvimento de recursos que permitam uma vantagem competitiva dentro das organizações.

Revista Ibero-Americana de Estratégia - RIAE, São Paulo, v. 8, n. 2, p. 04-30, jul./dez. 2009. 
Mário José Batista Franco \& Marisa Regina Reduto Santos Barbeira

Terra (2001) também desenvolveu uma abordagem em que, sob o ponto de vista estratégico, a gestão do conhecimento pode ser entendida a partir de sete dimensões: (1) o papel da administração; (2) cultura organizacional; (3) estrutura organizacional; (4) política e recursos humanos; (5) sistemas de informação; (6) mensuração dos resultados; e (7) relações externas.

A primeira dimensão, o papel da administração, refere-se ao papel indispensável desta na definição dos campos do conhecimento, na clarificação da estratégia organizacional e definição de metas e objectivos a atingir. A cultura organizacional deve estar condicionada ao desenvolvimento de inovação e optimização de todas as áreas da organização como uma estratégia dentro da mesma. As estruturas organizacionais estão relacionadas com o trabalho desenvolvido dentro das equipas multidisciplinares. A quarta dimensão, política e recursos humanos, está associada com a aquisição de conhecimento externo, por meio de novas contratações; e, interno, através da difusão e armazenamento de conhecimento na organização. O impacto que os sistemas de informação exercem sobre os mecanismos de criação, difusão e armazenamento de conhecimento faz deles uma dimensão fundamental para uma gestão estratégica do conhecimento. Destaca-se, também, a necessidade de mensuração dos resultados, principalmente na avaliação do capital intelectual. Por fim, as relações externas, através de relações com outras organizações e relacionamentos com os respectivos membros, sugerem uma estratégia organizacional.

McCann e Buckner (2004) identificaram ainda vários factores estratégicos: a forma como o conhecimento é avaliado, expresso, construído internamente e/ou adquirido externamente; partilhado internamente e/ou externamente; armazenado; e, aplicado para a criação e sustentação da estratégia organizacional.

Embora o conhecimento possa sempre ser partilhado, a forma como este é aplicado difere de pessoa para pessoa e situação para situação (Snyman \& Kruger, 2004). Estes autores abordam a gestão estratégica do conhecimento sob duas dimensões. A primeira consiste nas actividades críticas para a criação do conhecimento e inovação. A segunda dimensão consiste naqueles elementos que permitem ou influenciam as actividades de criação do conhecimento, tais como estratégia, política, tecnologia, cultura, entre outras.

Um sistema de gestão do conhecimento na organização envolve a interligação articulada e intencional dos seus membros de forma que, com base no conhecimento, seja possível compartilhar ideias e raciocínios a fim de estimular a colaboração e a cooperação visando à promoção do desempenho organizacional.

Nonaka e Takeuchi (1997) referem-se a "dispositivos" por onde o conhecimento individual é conectado e distribuído, difundindo-se em espiral na organização. Esses dispositivos têm a função

Revista Ibero-Americana de Estratégia - RIAE, São Paulo, v. 8, n. 2, p. 04-30, jul./dez. 2009. 
de promover uma interacção contínua e dinâmica que propicia a partilha e criação do conhecimento. Os referidos autores apresentam os seguintes promotores do conhecimento:

a) Socialização do conhecimento tácito em conhecimento tácito - processo em que são partilhadas as experiências individuais e colectivas;

b) Exteriorização do conhecimento tácito em conhecimento explícito - processo que procura tornar explícito e disponível em alguma forma de registo do conhecimento de cada um. A escrita é um exemplo dessa forma;

c) Combinação do conhecimento explícito em conhecimento explícito - processo em que as pessoas trocam e sintonizam conhecimentos por intermédio de formas externas, tais como relatórios, documentos e comunicação telefónica;

d) Internalização do conhecimento explícito em conhecimento tácito - processo em que cada colaborador vai aprender fazendo. Ocorre quando o mesmo consegue assimilar e aplicar o que aprendeu. Por exemplo, a reconfiguração de modelos mentais, a partilha de experiências através "da socialização, externalização e combinação".

Dessa forma, uma organização que promove a criação e a partilha de conhecimento, tanto tácito quanto explícito, pode ter melhores condições para actuar de forma dinâmica e estratégica em diversas áreas da sua actividade. Hope (2000) refere que muitas organizações percebem que o sucesso de longo prazo depende da acumulação e do uso produtivo baseados no conhecimento.

Finalmente, as actividades principais para um sistema de gestão do conhecimento prendemse com a geração (criação ou aquisição), codificação (ou armazenamento) e transferência do conhecimento. Porém, existem autores que defendem que os níveis de conhecimento das organizações são influenciados por quatro processos: criação do conhecimento, absorção de conhecimento público pertinente (externo), conhecimento transferido dentro da organização e disseminação no ambiente externo da organização do conhecimento pertencente à mesma. Cada um desses processos afecta o nível de conhecimento global da organização (Braunerhjelm, 2008; Fliaster \& Spiess, 2008; Franco \& Mariano, 2007; Marouf, 2007; Matusik, 2002; Weber \& Khademian, 2008).

Revista Ibero-Americana de Estratégia - RIAE, São Paulo, v. 8, n. 2, p. 04-30, jul./dez. 2009. 
Mário José Batista Franco \& Marisa Regina Reduto Santos Barbeira

\subsection{PROPOSTA DE UM MODELO CONCEPTUAL DE ANÁLISE}

A partir da revisão da literatura efectuada constata-se que a realização de redes estratégicas permitem, nos processos de relacionamento organizacional (intra e inter), diversas formas de cooperação e parcerias entre as organizações (Nonaka \& Takeuchi, 1997). Simultaneamente, o desenvolvimento de redes estratégicas pode ser o método mais rápido e eficaz para adquirir e partilhar o conhecimento. A transferência de conhecimento pode ser vista como um instrumento fulcral nos relacionamentos entre e intraorganizacionais.

Relativamente às redes estratégicas estabelecidas nas organizações, a aquisição de conhecimentos decorre da integração do conhecimento proveniente de fontes externas (nomeadamente de outras organizações) e da sua integração na base de conhecimentos existentes na organização (Grant, 1991).

Nesse sentido, Levinson e Asahi (1995) identificaram quatro passos que podem ocorrer dentro de um processo de rede interorganizacional. O primeiro passo envolve a participação de uma organização no meio envolvente para a identificação de novos conhecimentos (técnico, cultural ou de gestão). O segundo passo prende-se com a transferência de conhecimento. Uma vez a informação ter sido identificada, é necessário transmiti-la ao longo de um caminho previamente seleccionado. Essas ligações estruturais influenciam não só a capacidade para a identificação de novo conhecimento, mas também a sua transmissão. $O$ terceiro passo envolve a utilização de processos de conhecimento. Após a transferência do conhecimento numa rede, este conhecimento é utilizado para alcançar um resultado. Por último, o quarto passo que pode ocorrer na rede interorganizacional é a institucionalização do conhecimento.

As redes interorganizacionais têm sido apresentadas como formas particularmente úteis para as organizações aprenderem umas com as outras implementando medidas inovadoras em termos estratégicos de competitividade (Kogut, 1988). De facto, as redes estratégicas estabelecidas dentro das organizações constituem vectores fundamentais da comunicação, da interacção, da partilha e da difusão do conhecimento (Rolo, 2005).

Atendendo ao supracitado, é proposto um modelo conceptual (Figura 1), que permite perspectivar o papel de um sistema de gestão do conhecimento como fomentador das redes intra e interorganizacionais, e estas como uma estratégia para a partilha de conhecimento.

Revista Ibero-Americana de Estratégia - RIAE, São Paulo, v. 8, n. 2, p. 04-30, jul./dez. 2009. 
Figura 1- Modelo conceptual de análise.

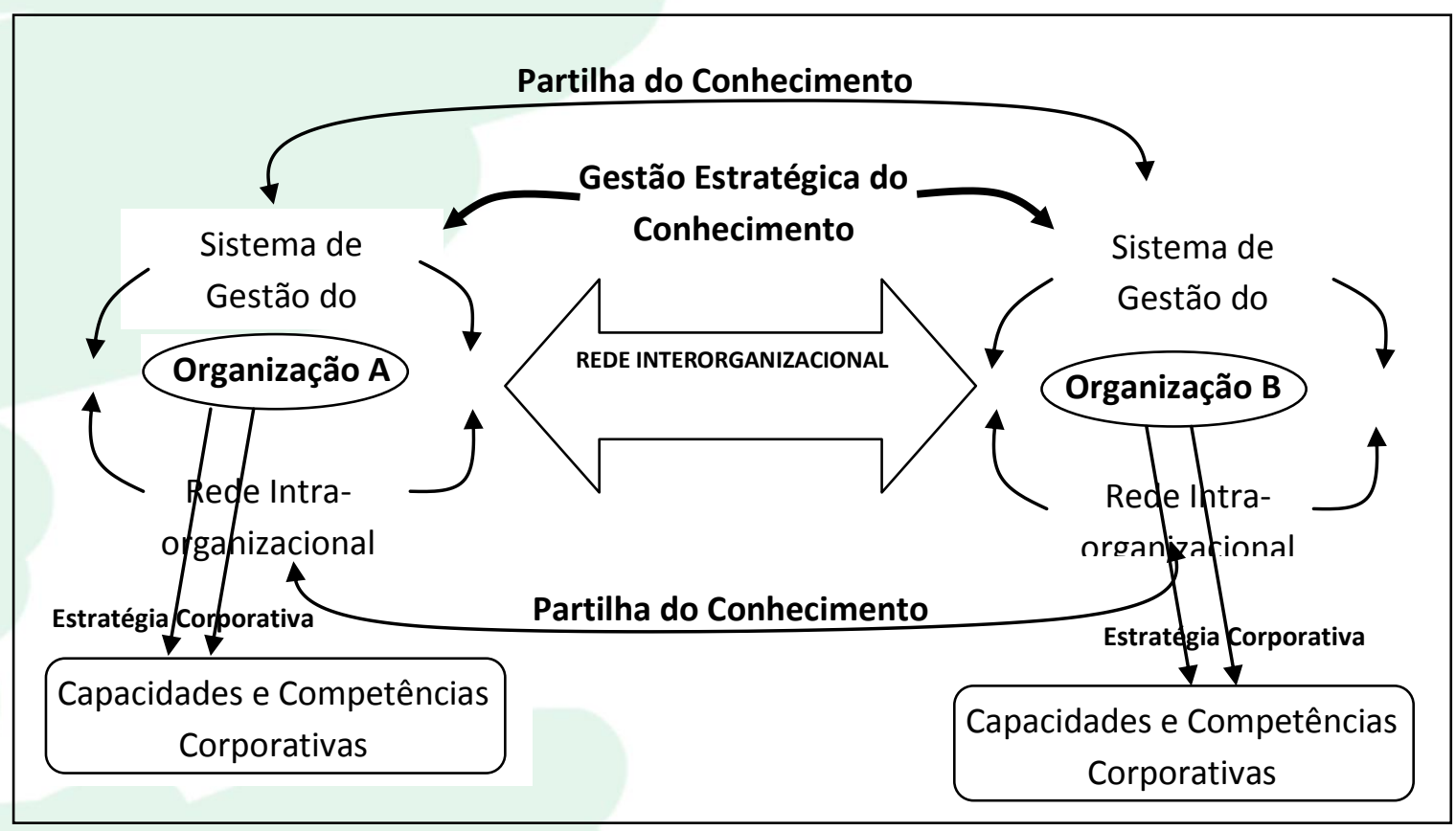

Fonte: Elaborado pelos autores.

O modelo assenta num sistema de gestão do conhecimento como fomentador de redes organizacionais. Para isso, existe o envolvimento e a participação de duas organizações (A) e (B), que se relacionam através da transferência de conhecimentos (redes intraorganizacionais). Por outro lado, de acordo com os seus interesses, cada organização possui o seu próprio sistema com ênfases e focos específicos. Dentro de cada organização também podem ocorrer, através dos seus membros, ligações estruturais que influenciam não só a capacidade para a identificação de novo conhecimento, mas também a sua transmissão. A transferência do conhecimento nesse tipo de rede pode ser utilizado para implementar medidas estratégicas, que estimulem e promovam a relação entre as organizações no contexto da aprendizagem e que, simultaneamente, funcionem como uma estratégia para a partilha do conhecimento.

Refira-se ainda, de acordo com Felman, Gertler e Wolfe (2006) e Fliaster e Spiess (2008), que as organizações diferem no seu conhecimento interno, nas suas práticas e nas suas capacidades. A verdadeira contribuição das redes, para as organizações integrantes, é o desenvolvimento de capacidades e competências organizacionais específicas, incluindo também aspectos que se refiram ao melhoramento de processos (administrativos e de produção) e de domínio tecnológico. As redes de ligações entre unidades permitem, às organizações, aceder a novos conhecimentos uns dos outros

Revista Ibero-Americana de Estratégia - RIAE, São Paulo, v. 8, n. 2, p. 04-30, jul./dez. 2009. 
Mário José Batista Franco \& Marisa Regina Reduto Santos Barbeira

e poder aumentar a sua eficiência de custo através da disseminação das "melhores práticas" entre as organizações. A centralidade de uma unidade na rede pode determinar o acesso a conhecimento diferente, afectando assim a sua habilidade para reconhecer e responder a novas oportunidades de mercado.

Realçando a transferência do conhecimento, as redes, visando estandardizar algumas formas de acção, procuram estabelecer um relacionamento forte, o que conduz, assim, a um reforço do compromisso que cada unidade possui na rede. Mas, os relacionamentos, nesse segmento, podem ser fortes ou fracos, dependendo, segundo Fliaster e Spiess (2008), Granovetter (1973, 1982), Marouf (2007) e Nelson $(1986,1989)$ de as ligações ou contactos serem formais ou informais, frequentes ou não frequentes ou puramente utilitários.

A capacidade de assimilação e de difusão do conhecimento torna a rede robusta e consolida as uniões e os seus vértices de ligação, proporcionando, desse modo, a criatividade e a inovação dos produtos e/ou serviços, marcando uma posição sólida, credível e duradoura no meio organizacional.

O modelo proposto sugere ainda que, para se ligar um sistema de conhecimento com a estratégia organizacional, torna-se necessário estabelecer uma ligação ao conceito de estratégia corporativa (Camargos \& Dias, 2003; Miles \& Snow; 1978). Como refere Gupta (1999), a estratégia corporativa é o nível mais elevado da estratégia onde se tratam aspectos mais amplos, como, por exemplo, a forma de explorar as sinergias entre unidades organizacionais. Nesse contexto, as redes interorganizacionais são vistas como um tipo de estratégia corporativa, em que um relacionamento entre organizações permite partilhar actividades, capacidades e competências corporativas.

As redes organizacionais não só funcionam como canais de comunicação, mas também como canais de "alimentação" das organizações, não só em nível de matérias-primas e de recursos aos mais variados níveis, como também em nível ideológico, como importante forma de difusão e partilha de ideias e tendências.

A transmissão formal dos conhecimentos mediante os grupos ou equipas de trabalho, bem como o intercâmbio de informação que se produz na organização são, portanto, aspectos considerados como importantes para o êxito da rede. A estratégia, as pessoas envolvidas, assim como o desenvolvimento de uma estrutura organizacional que condiciona o funcionamento das empresas, pessoas e grupos, são também factores que podem condicionar o processo de rede.

Por último, uma rede é composta por todas as unidades organizacionais e sociais de um tipo específico de relacionamento. Segundo Chisholm (1996), uma rede pode desenvolver-se em vários níveis. Esses níveis incluem relações intergrupos, interdepartamentos e interorganizacionais, mas

Revista Ibero-Americana de Estratégia - RIAE, São Paulo, v. 8, n. 2, p. 04-30, jul./dez. 2009. 
sempre tendo como resultado a partilha do recurso mais valioso que uma organização pode e deve aceder: o conhecimento.

\section{CONSIDERAÇÕES FINAIS}

A potencialidade das redes estratégicas organizacionais em gerar e partilhar novos conhecimentos tem vindo a ser evidenciada no campo organizacional. O conhecimento nasce em um nível individual, sendo ampliado pela dinâmica da interacção (socialização do conhecimento) para um nível organizacional e, posteriormente, para um nível interorganizacional. Desse modo, as redes estratégicas promovem a interacção, o dinamismo, a confiança e, sobretudo, o relacionamento dentro e entre as organizações. Dessa forma, é pertinente a aplicação da gestão estratégica do conhecimento e de um sistema de gestão do conhecimento nas organizações, já que, estrategicamente, servem como mecanismos de produtividade, eficiência e excelência organizacional. Por outro lado, o conhecimento proveniente de redes interorganizacionais actua não apenas como apoio para as actividades organizacionais, mas serve também como fonte de informação para o processo de decisão e a melhoria do desempenho.

De facto, as redes interorganizacionais criam e desenvolvem um novo conhecimento, novas formas de interagir, reforçando as forças com vista a atingirem maiores proveitos como máximo para conquistar níveis de excelência da própria rede e dos elementos que a compõem. Todavia, apesar de algumas investigações terem referido a importância das relações interorganizacionais e mostrado este reconhecimento, existem ainda poucos estudos sobre o impacto das redes interorganizacionais na partilha do conhecimento. Assim, um dos contributos deste estudo foi a identificação de alguns elementos básicos necessários para conduzir futuras investigações sobre a importância das redes na transferência do conhecimento dentro e entre organizações e como um sistema de gestão do conhecimento pode ser um importante fomentador de tais relacionamentos.

Com este trabalho conclui-se, ainda, que para as organizações se tornarem competitivas e alcançarem sucesso necessitam criar e gerar conhecimento e, este último, pode e deve ser assimilado e transferido entre os vários actores e/ou agentes mediante o estabelecimento de redes intra e interorganizacionais.

Tendo por base essas considerações, elaborou-se um modelo conceptual para a análise das redes interorganizacionais que contemplou vários aspectos, tais como a gestão estratégica do

Revista Ibero-Americana de Estratégia - RIAE, São Paulo, v. 8, n. 2, p. 04-30, jul./dez. 2009. 
conhecimento, a gestão corporativa e, basicamente, como tais redes podem ser fomentadas por um sistema de gestão do conhecimento, tudo isso originando como resultado final a partilha do conhecimento. Em suma, propôs-se que, para analisar as redes inteorganizacionais, deve-se ter em atenção o contexto em que as organizações operam, as estratégias que podem ser adoptadas, os motivos e os benefícios de tais redes organizacionais.

Como limitação deste estudo, aponta-se o facto do modelo aqui proposto não ter sido testado empiricamente e não terem sido expostos os indicadores para medir e avaliar as várias dimensões que o constitui. Desse modo, sugere-se para futuras investigações, nesta área do saber, que o modelo conceptual de referência aqui apresentado seja validado empiricamente em diferentes tipos de organizações.

\section{Agradecimentos}

Os autores agradecem os comentários e as sugestões dadas pelos dois revisores anónimos que avaliaram o presente artigo, nomeadamente, um deles que contribuiu fortemente na construção do modelo conceptual de análise proposto.

\section{REFERÊNCIAS}

Aaker, D. A. (1992). Strategic market management. New York: John Wiley \& Sons.

Azevedo, A. L. (2000). A emergência de redes de empresas. Recuperado em 31 de novembro, 2007, de

http://www.spi.pt/documents/books/ecommerce/cenmn/acesso_ao_conteudo_integral/capitulos/1 .3/cap_actual.htm

Balestrin, A., \& Vargas, L. M. (1998). Monitoramento do ambiente concorrencial na indústria metal-mecânica da região de Caxias do Sul. Anais do Encontro da Associação Nacional de PósGraduação e Pesquisa em Administração, 22. Rio de Janeiro: ANPAD.

Balestrin, A., \& Vargas, L. M. (2002). Evidências teóricas para a compreensão das redes interorganizacionais [CD-ROM]. Anais do Encontro Nacional de Estudos Organizacionais, 2. Rio de Janeiro: ANPAD.

Revista Ibero-Americana de Estratégia - RIAE, São Paulo, v. 8, n. 2, p. 04-30, jul./dez. 2009. 
Bloodgood, J. M., \& Salisbury, W. D. (2001). Understanding the influence of organizational change strategies on information technology and knowledge management strategies. Decision Support Systems, 31(1), 55- 69.

http://dx.doi.org/10.1016/S0167-9236(00)00119-6

Braunerhjelm, P. (2008). Specialization of regions and universities: the new versus the old. Industry and Innovation, 15(3), 253-275.

http://dx.doi.org/10.1080/13662710802040853

Camargos, M. A., \& Dias, A. T. (2003). Estratégia, administração estratégica e estratégia corporativa: uma síntese teórica. Caderno de Pesquisas em Administração, 10(1), 27-39.

Cassiman, B., \& Veugelers, R. (2002). R\&D cooperation and spillovers: some empirical evidence from Belgium. American Economic Review, 92(4), 1169-1184.

http://dx.doi.org/10.1257/00028280260344704

Chisholm, R. F. (1996). On the meaning of networks. Group \& Organization Management, 21(2), 216-236.

http://dx.doi.org/10.1177/1059601196212006

Contractor, F., \& Lorange, P. (1988). Why should firms cooperate?. In F. Contractor \& P. Lorange (Eds), Cooperative strategies in international business (pp. 3-18). Lexington, MA: Lexington Books.

Coviello, N. (2006). The network dynamics of international new ventures. Journal of international Business Studies, 37, 713-731.

Cozzarin, B. P., \& Percival, J. C. (2006). Complementarities between organizational strategies and innovation. Economics of Innovation and New Technology, 15(3)3, 195-217.

http://dx.doi.org/10.1080/10438590500222691

Davenport, T. H., \& Prusak, L. (1998). Conhecimento empresarial: como as organizações gerenciam o seu capital intelectual. Rio de Janeiro: Campus.

Drucker, P. F. (1993). Sociedade pós-capitalista. São Paulo: Pioneira.

Dussauge, P., \& Garrette, B. (1995). Determinants of success in international strategic alliances: evidence from the global aerospace industry. Journal of International Business Studies, 26, 505528. http://dx.doi.org/10.1057/palgrave.jibs.8490848

Eiriz, V. (2004). Dinâmica de relacionamento entre redes inter - organizacionais. Inovação Organizacional, 2, 121-153.

Revista Ibero-Americana de Estratégia - RIAE, São Paulo, v. 8, n. 2, p. 04-30, jul./dez. 2009. 
Mário José Batista Franco \& Marisa Regina Reduto Santos Barbeira

Faggion, G. A., Balestrin, A., \& Weyh, C. (2002). Geração de conhecimento e inteligência estratégica no universo das redes interorganizacionais. Revista Inteligência Empresarial, 12, 5765 .

Faulkner, D. (1992). Strategic alliances: cooperation for competition. In D. Faulkner \& G. Johnson (Eds), The challenge of strategic management (pp.119-146). London: Kogan Page, 1992.

Felman, M., Gertler, M., \& Wolfe, D. (2006). University technology transfer and national systems of innovation: introduction to the special issue of industry and innovation, Industry and Innovation, 13(4), 359-370.

http://dx.doi.org/10.1080/13662710601035781

Fleury, M. T., \& Oliveira Jr., M. M. (2001). Gestão estratégica do conhecimento. São Paulo: Atlas.

Fliaster, A., \& Spiess, J. (2008). Knowledge mobilization through social ties: the cost-benefit analysis. Schmalenbach Business Review, 60, 99-117.

Forbes, N., \& Wield, D. (2008). Innovation dynamics in catch-up firms: process, product and proprietary capabilities for development. Industry and Innovation, 15(1), 69-92.

http://dx.doi.org/10.1080/13662710701850741

Franco, M., \& Mariano, S. (2007). Information technology repositories and knowledge management processes. Journal of Information and Knowledge Management Systems, 37(4), 440-451.

Franco, M. (2001). O processo de cooperação nas empresas portuguesas: formação, implementação e desenvolvimento. Tese de Doutorado, Universidade da Beira Interior, Covilhã, Portugal.

Franco, M. (2003). Collaboration among SMEs as a mechanism for innovation: an empirical study. New England Journal of Entrepreneurship, 6(1), 23-32.

Fritsch, M. (2004). Cooperation and the efficiency of regional R\&D activities. Cambridge Journal of Economics, 28(6), 829-846.

http://dx.doi.org/10.1093/cje/beh039

Granovetter, M. (1973). The strength of weak ties. American Journal of Sociology, 78(6), 13601380.

Granovetter, M. (1982). The strength of weak ties: a network theory revisited. In P. V. Marsden \& N. Lin, Social structure and network analysis (pp.105-131). Beverly Hills: Sage Publications. http://dx.doi.org/10.1086/225469

Revista Ibero-Americana de Estratégia - RIAE, São Paulo, v. 8, n. 2, p. 04-30, jul./dez. 2009. 
Grant, R. M. (1991). The resource-based theory of competitive advantage: implications for strategy formulation. California Management Review, 33(3), 114-134.

Grant, R. M. (2006). The knowledge-based view of the firm. In D. Faulkner \& A. Campbell, Strategy: a strategy overview and competitive strategy (pp. 203-227). Oxford: University Press.

Gray, P. H., \& Meister, D. B. (2006). Knowledge sourcing methods. Information \& Management, 43(2), 142-156.

http://dx.doi.org/10.1016/j.im.2005.03.002

Gupta, A. K. (1999). Estratégia das unidades de negócio: gerenciando um único negócio. In L. Fahey, \& R. M. Randall, MBA curso prático: estratégia (pp.100-125) (2a ed). Rio de Janeiro: Campus.

Häkansson, H., \& Ford, D. (2002). How should companies interact in business networks? Journal of Business Research, 55(2), 133-139.

http://dx.doi.org/10.1016/S0148-2963(00)00148-X

Håkansson, H, \& Snehota, I. (1989). No business is an island: the network concept of business strategy. Scandinavian Journal of Management, 5(3), 187-200.

http://dx.doi.org/10.1016/0956-5221(89)90026-2

Hansen, M. T., Nohria, N., \& Tierney, T. (1999). What's your strategy for managing knowledge? Harvard Business Review, 77(2), 106-116.

Hax, A. C., \& Majluf, N. S. (1988). The concept of strategy and the strategy formation process. Interfaces, 18(3), 99-109.

$\underline{\text { http://dx.doi.org/10.1287/inte.18.3.99 }}$

Hitt, M. A., Ireland, R. D., \& Hoskisson R. E. (2001). Direct and moderating effects of human capital on strategy and performance in professional service firms: a resource - based perspective. Academy of Management Journal, 44(1), 13-28.

http://dx.doi.org/10.2307/3069334

Hoang, H., \& Antoncic, B. (2003). Network-based research in entrepreneurship: a critical review. Journal of Business Venturing; 18(2), 165-187. http://dx.doi.org/10.1016/S0883-9026(02)00081-2

Hope, J. (2000). Competindo na terceira onda: os 10 mandamentos da era da informação. Rio de Janeiro: Campus.

Huang, H. C., \& Chang, C. W. (2008). Embedded ties and the acquisition of competitive advantage. Journal of Intellectual Capital, 9(1), 105-121.

http://dx.doi.org/10.1108/14691930810845830

Revista Ibero-Americana de Estratégia - RIAE, São Paulo, v. 8, n. 2, p. 04-30, jul./dez. 2009. 
Mário José Batista Franco \& Marisa Regina Reduto Santos Barbeira

Inkpen, A. C., \& Beamish, P. W. (1997). Knowledge, bargaining power, and the instability of international joint ventures. Academy of Management Journal, 22(1), 177-202.

Inkpen, A. C., \& Tsang (2005). Creating knowledge through collaboration. California Management Review, 39(1), 123-140.

Johanson, J., \& Mattsson, L. (1987). Interorganizational relations in industrial systems: a network approach compared with the transaction cost approach. International Studies of Management \& Organization, 17(1), 34-48.

Johnson, G., \& Scholes, K. (1999). Exploring corporate strategy (5 $5^{\text {th }}$ ed). New York: Prentice Hall.

Kogut, B. (1988). Joint ventures: theoretical and empirical perspectives. Strategic Management Journal, 9, 319-332. http://dx.doi.org/10.1002/smj.4250090403

Krog, G. (1998). Care in knowledge creation. California Management Review, 40(3), 133-153.

Lesca, H., Freitas H., \& Cunha M. V. M. (1996). Como dar um senso útil às informações dispersas para facilitar as decisões e acções dos dirigentes - o problema crucial da inteligência competitiva através da construção de um puzzle. Revista Eletrônica de Administração, 2(2), 11-19.

Levinson, N. S., \& Asahi, M. (1995). Cross-national alliances and interorganizational learning. Organizational Dynamics, 24(2), 50-62.

http://dx.doi.org/10.1016/0090-2616(95)90071-3

Lorange, P., \& Roos, A. (1996). Cooperative strategies in international business: joint ventures and technology partnerships between firms. New York: Lexington Books.

Lorenz, E. H. (1992). Trust, community and co-operation: toward a theory of industrial districts. In M. Storper \& A. J. Scott (Eds.), Pathways to industrialization and regional development (pp.175-182). London: Routledge.

Loureiro, J. L. (2003). Gestão do conhecimento. Lisboa: Centro Atlântico, Lisboa.

Macnabb, A. I., \& O’Neill, K. E. (1996). Small firm, large firm collaboration for survival and growth in the world market: the case of Schrader Electronics Ltd. Proceedings of the International Council for Small Business World Conference, 41 (pp. 1-11). Stockholm, SE.

Marouf, L. N. (2007). Social networks and knowledge sharing in organizations: a case study. Journal of Knowledge Management, 11(6), 110-125. http://dx.doi.org/10.1108/13673270710832208

Revista Ibero-Americana de Estratégia - RIAE, São Paulo, v. 8, n. 2, p. 04-30, jul./dez. 2009. 
Márquez-García, A. M., Hernández-Ortiz, M. J., Vallejo-Martos, M. C., \& Cañas-Lozano, L. (1988). The role of trust in cooperation relationships. Proceedings of the International Conference of the Association of Management and Business Economics, 7 (pp. 307-318). Istanbul, TR.

Mason, J. C. (1993). Strategic alliances: partnering for success. Management Review, 82(5), 10-15.

Matusik, S. F. (2002). Managing public and private firm knowledge within the context of flexible firm boundaries. In C. W. Choo \& N. Bontis (Eds.), The strategic management of intellectual capital and organizational knowledge (pp. 605-617). New York: Oxford University Press.

McCann, J. E., \& Buckner, M. (2004). Strategically integrating knowledge management initiatives. Journal of Knowledge Management, 8(1), 1-19. http://dx.doi.org/10.1108/13673270410523907

Miles, R. E., \& Snow, C. C. (1978). Organizational strategy, structure and process. New York: McGraw-Hill.

Mintzberg, H., Ahlstrand, B., \& Lampel J. (2000). Safari de estratégia: um roteiro pela selva do planejamento estratégico. Porto Alegre: Bookman.

http://dx.doi.org/10.1108/00251749710173715

Morrison, M., \& Mezentseff, L. (1997). Learning alliances - a new dimension of strategic alliances. Management Decision, 35(5), 351-357.

Moura, A. (2001). Algumas referências básicas para a gestão do conhecimento. Porto Alegre: Afrontamento.

Murray, J. Y. (1995). Patterns in domestic vs. international strategic alliances: an investigation of U.S. multinational firms. Multinational Business Review, 3(2), 7-16.

Nadler, D. A., \& Tushman, M. L. (2000). A organização do futuro: as ligações mais importantes do século XX e os próximos desafios que levarão ao novo desenho da empresa. HSM Management, $18,58-66$.

Nelson, R. E. (1986). Social networks and organizational intervention: insights from an area-wide labor-management committee. Journal of Applied Behavioral Science, 22, 65-76.

http://dx.doi.org/10.1177/002188638602200109

Nelson, R. E. (1989). The strength of strong ties: social networks and intergroup conflict in organizations. Academy of Management Journal, 32(2), 377-401.

http://dx.doi.org/10.2307/256367

Revista Ibero-Americana de Estratégia - RIAE, São Paulo, v. 8, n. 2, p. 04-30, jul./dez. 2009. 
Mário José Batista Franco \& Marisa Regina Reduto Santos Barbeira

Nonaka, I., \& Takeuchi, H. (1997). Criação de conhecimento na empresa: como as empresas japonesas geram a dinâmica da inovação. Rio de Janeiro: Campus.

Parkhe, A., Wasserman, S., \& Ralston, D. (2006). New frontiers in network theory development. Academy of Management Review, 31(3), 560-568.

http://dx.doi.org/10.5465/AMR.2006.21318917

Percival, J. C., \& Cozzarin, B. P. (2008). Complementarities affecting the returns to innovation. Industry and Innovation, 15(4), 371-392.

http://dx.doi.org/10.1080/13662710802273249

Quinn, J. B. (1996). Empresas muito mais inteligentes: como integrar recursos intelectuais, produtos e serviços de formas úteis e dinâmicas, adicionando valores a seus clientes. São Paulo: Makron Books.

Rolo, T. (2005). As relações inter-organizacionais - o caso da indústria automóvel. Trabalhos Apresentados no Seminário sobre Redes Organizacionais. Porto, PR.

Root, F. R. (1988). Some taxonomies of international cooperative arrangements. New York: Lexington Books.

Seerano, A., \& Fialho, C. (2003). Gestão do conhecimento - o movo paradigma das organizações. Lisboa: FCA.

Schmiedeberg, C. (2008). Complementarities of innovation activities: an empirical analysis of the German manufacturing sector. Research Policy, 37(9), 1492-1503.

http://dx.doi.org/10.1016/j.respol.2008.07.008

Sher, J. P., \& Lee, C. V. (2004). Information technology as a facilitator for enhancing dynamic capabilities through knowledge managements. Information and Management, 41(8), 933-945. http://dx.doi.org/10.1016/j.im.2003.06.004

Silva, R. V., \& Neves, A. (2003). Gestão de empresas - na era do conhecimento. Lisboa: Sílabo.

Snyman, R., \& Kruger, C. J. 2004. The interdependency between strategic management and strategic knowledge management. Journal of Knowledge Management, 8(1), 47-63.

http://dx.doi.org/10.1108/13673270410523871

Soo, C. (2002). Knowledge management: philosophy, process, pitfalls, and performance. California Management Review, 44(4), 129-151.

Stafford, E. R. (1994). Using co-operative strategies to make alliances work. Long Range Planning, 27(3), 64-74. http://dx.doi.org/10.1016/0024-6301(94)90191-0

Revista Ibero-Americana de Estratégia - RIAE, São Paulo, v. 8, n. 2, p. 04-30, jul./dez. 2009. 
Suire, R., \& Vicente, J. (2008). Theórie économique des clusters et management des réseaux d'entreprises innovantes. Revue Française de Gestion, 184, 119-136.

Sveiby, K. E. (1998). A nova riqueza das organizações: gerenciando e avaliando patrimónios de conhecimento. Rio de Janeiro: Campus.

Teece, D. J. (1998). Capturing value from knowledge assets: the new economy, markets for knowhow, and intangible assets. California Management Review, 40(3), 52-79.

Teece, D. J. (2000). Strategies for managing knowledge assets: the role of firm structure and industrial context. Long Range Planning, 33(1), 35-36.

http://dx.doi.org/10.1016/S0024-6301(99)00117-X

Terra, J. C. (2001). Gestão do conhecimento: o grande desafio empresarial: uma abordagem baseada no aprendizado e na criatividade. São Paulo: Negócio.

Thompson Jr., R. A. A., \& Strickland III, A. J. (2000). Planejamento estratégico: elaboração, implementação e execução. São Paulo: Cengage Learning.

Tidd, J., Bessant, J., \& Pavitt, K. (2003). Gestão da inovação - integração das mudanças tecnológicas de mercado e organizacionais. Lisboa: Monitor - Projectos.

Weber, E. P., \& Khademian, A. M. (2008). Wicked problems, knowledge challenges, and collaborative capacity builders in network settings. Public Administration Review, 68(2), 334349.

http://dx.doi.org/10.1111/j.1540-6210.2007.00866.x

Yeung, H. W. (1994). Critical reviews of geographical perspectives on business organizations and the organization of production: towards a network approach. Progress in Human Geography, 18(4), 460-490.

http://dx.doi.org/10.1177/030913259401800403

Yoshino, M. Y., \& Rangan, U. S. (1995). Strategic alliances: an entrepreneurial approach to globalization. Boston: Harvard Business Press.

Zack, M. H. (1999). Developing a knowledge strategy. California Management Review, 41(3), 125145.

Zinn, J. S., Proença, J., \& Rosko, M. D. (1997). Organizational and environmental factors in hospital alliance membership and contract management: a resource-dependence perspective. Hospital \& Health Services Administration, 42(1), 67-86.

Revista Ibero-Americana de Estratégia - RIAE, São Paulo, v. 8, n. 2, p. 04-30, jul./dez. 2009. 
Recebido: 05/05/09

Aprovado: 30/07/09

Revista Ibero-Americana de Estratégia - RIAE, São Paulo, v. 8, n. 2, p. 04-30, jul./dez. 2009. 